\title{
Apgar Score at Twenty Minutes
}

National Cancer Institute

\section{Source}

National Cancer Institute. Apgar Score at Twenty Minutes. NCI Thesaurus. Code C98820.

The numerical sum between zero and ten that is computed from individual values of zero

to two that are assigned to five different areas of interest in newborn screenings at twenty minutes after birth. These areas are breathing, muscle tone, pulse rate, reflex irritability and skin color. 